\title{
Acetylcholinesterase and butyrylcholinesterase activities in cerebrospinal fluid from different levels of the neuraxis of patients with dementia of the Alzheimer type
}

\author{
Margaret E Appleyard, Brendan McDonald
}

\begin{abstract}
Acetylcholinesterase (AChE) and butyrylcholinesterase (BuChE) activities of cerebrospinal fluid (CSF) collected post mortem from the lateral ventricles, cisterna magna, and lumbar regions of the spinal cord of patients with a histologically confirmed diagnosis of Alzheimer's disease were compared with those of normal, age matched control patients, patients with dementia of nonAlzheimer aetiology, and patients with non-dementing neurological disorders. The AChE activity of the ventricular CSF of patients with Alzheimer's disease was $48 \%$ lower $(p<0.005)$ than that of age matched controls or patients with other types of dementia, and the AChE activity of CSF sampled from the basal cistern was $40 \%$ lower $(p<0.005)$ in patients with Alzheimer's disease. There were no significant differences between the $\mathrm{AChE}$ activity in Alzheimer's disease and control patients in CSF collected from the lumbar cistern. AChE activity was lower in CSF sampled from the basal and lumbar cistern of patients with dementia of nonAlzheimer aetiology, while ventricular activity was in the normal range. BuChE activity in ventricular CSF of Alzheimer's disease patients was $41 \%$ lower than normal $(p<0.05)$ and in the normal range in all other samples. The secretion of $\mathrm{AChE}$ from forebrain and hindbrain regions is reduced in Alzheimer's disease patients, leading to decreased ventricular and cisternal levels of the enzyme. Secretion from more caudal regions of the central nervous system seems to be unaffected by the disease, resulting in $\mathrm{AChE}$ in the lumbar CSF of patients with Alzheimer's disease being in the control range. Such altered secretion of $\mathrm{AChE}$ in the brain could have profound implications not only for cholinergic transmission in these patients but also for the proposed noncholinergic modulatory actions of $\mathrm{AChE}$.
\end{abstract}

Neuroanatomical Pharmacology, Oxford OX1 3QT

Brendan McDonald

Correspondence to: Dr M E Appleyard, Department of Physiology, Royal Free Hospital Schoo of Medicine, Rowland Hill Street, London NW3 2PJ.

Received 5 July 1991 and in revised form 12 November 1991 and 2 March 1992

Accepted 10 March 1992 (F Neurol, Neurosurg Psychiatry 1992;55:1074-1078)

A deficit of acetylcholinesterase (EC $3 \cdot 1 \cdot 1 \cdot 7$; $\mathrm{AChE}$ ) activity in several brain regions is a characteristic feature of Alzheimer's disease. ${ }^{1}$ Acetylcholinesterase is secreted from several brain regions of animals on electrical and drug stimulation $^{2}$ and is present in cerebrospinal fluid (CSF). Nevertheless, attempts to determine whether the lower activity of AChE in brain tissue found in patients with Alzheimer's disease is reflected in lower activity of AChE in CSF have proved inconclusive. Of 11 age matched studies utilising assay methods specific for $\mathrm{AChE}$, five found lower $\mathrm{AChE}$ activity in lumbar CSF of patients with Alzheimer's disease ${ }^{3-7}$ and the remainder found no change compared with control patients. ${ }^{8-13}$ Most of these studies had, however, relied solely on clinical diagnoses of their patients, but a definitive diagnosis of Alzheimer's disease is possible only after histological examination of brain material. ${ }^{14}$ Inclusion of patients with dementia of non-Alzheimer aetiology in some of these studies may help to explain the conflicting results.

A previous study from our laboratory ${ }^{15}$ that attempted to resolve this issue found that AChE activity in lumbar CSF (obtained during life) from patients with a clinical diagnosis of Alzheimer's disease, confirmed by histological examination at brain biopsy, were not significantly different from those found in age matched controls. ${ }^{15}$ In contrast, in a companion study AChE activity in ventricular CSF (obtained post mortem) were found to be significantly lower in patients with a histologically confirmed diagnosis of Alzheimer's disease, than in age matched controls. ${ }^{16}$ These conflicting results could be explained if the decreased activity found in ventricular CSF of patients with Alzheimer's disease were masked in lumbar CSF by additional AChE secreted from brain structures caudal to the ventricular system. Indeed, the lack of a rostrocaudal gradient of AChE activity indicates a diffuse origin of the AChE in lumbar CSF. The lower activity of AChE from the ventricular CSF of patients with Alzheimer's disease may, however, have been a postmortem artefact. In an attempt to distinguish between these two possibilities we have measured the AChE activity in samples of ventricular, basal, and lumbar cisternal CSF obtained from the same patients at postmortem examination.

Our previous study also found that butyryl- 
Methods

PATIENTS

Samples of CSF were collected at necropsy from 21 control subjects, 26 patients with dementia of the Alzheimer type, 12 patients with dementia of non-Alzheimer aetiology, and five patients with non-dementing neurological diseases. The ages and the delays between death and postmortem examination were similar in all the groups of patients (table 1). Some patients in each group were taking centrally acting drugs immediately before death, the most commonly prescribed drugs being benzodiazepines and chloral hydrate.

Diagnosis of Alzheimer's disease was made from a clinical history of progressive dementia before death and the presence of the characteristic neuropathological features of the disease at histological examination of brain tissue postmortem according to Khachaturian criteria. ${ }^{14}$ All patients included in this group had no clinical or histological signs of any other neurological disorder. The group included four patients with presenile Alzheimer's disease (onset before age 65), two of whom suffered from Down's syndrome. Patients with mixed Alzheimer and other pathology were excluded from the study.

Patients with dementia of non-Alzheimer aetiology also had a clinical history of dementia, but postmortem examination confirmed that this was due to causes other than Alzheimer's disease. Patients in this group included four patients with multi-infarct dementia, three patients with encephalopathies (one hepatic, two secondary to renal failure), one patient with striatonigral degeneration, and one patient with Pick's disease.

Age matched control patients had no clinical history of dementia or any other neurological disease and showed only age related changes on postmortem examination of the brain.

COLLECTION OF CSF

Samples of ventricular, cisternal, and lumbar CSF were collected at necropsy. However, it was not possible to collect all three fluids for every patient.

After the cranial cavity was opened, CSF was aspirated (using sterile disposable plastic pasteur pipettes) from the level of the basal cistern with the exposed brain still in situ. Ventricular CSF was obtained, after the brain was removed, by bisecting the forebrain in the midline sagittal plane, thereby exposing the lateral ventricles, from which the CSF was aspirated. CSF was collected from the lumbar cistern by dissection of lumbar vertebral bodies, thereby exposing the dura, through

Table 1 Mean (SE) age at death and delay to postmortem examination of patients studied

\begin{tabular}{lcll}
\hline Patients & No & Age (years) & Delay (hours) \\
\hline Controls & 21 & $76 \cdot 0(1 \cdot 9)$ & $57 \cdot 0(5 \cdot 2)$ \\
Alzheimer's disease & 26 & $77 \cdot 9(2 \cdot 0)$ & $49 \cdot 6(5 \cdot 0)$ \\
Senile (onset after age 65) & 22 & $81 \cdot 3(1 \cdot 1)$ & $51 \cdot 7(5 \cdot 5)$ \\
Presenile (onset before age 65) & 2 & $56 \cdot 0$ & $25 \cdot 0$ \\
With Down's syndrome & 2 & $62 \cdot 0$ & $42 \cdot 0$ \\
Other types of dementia & 12 & $72 \cdot 2(2 \cdot 7)$ & $68 \cdot 4(7 \cdot 7)$ \\
Other neurological diseases & 5 & $77 \cdot 2(3 \cdot 6)$ & $49 \cdot 3(6 \cdot 4)$ \\
\hline
\end{tabular}

which CSF was withdrawn after direct puncture. The CSF samples were then centrifuged at $3000 \mathrm{~g}$ for 30 minutes at $4^{\circ} \mathrm{C}$, the supernatant aliquoted, snap frozen, and stored at $-70^{\circ} \mathrm{C}$ for up to one year. Any samples that were visibly contaminated with blood were discarded.

A gross assessment of ventricular size was made during the necropsy and also after brain fixation. The degree of ventricular enlargement was graded as mild, moderate, or severe.

\section{BIOCHEMICAL ANALYSIS}

All biochemical analyses were performed in triplicate on coded samples without prior knowledge of the diagnosis.

Cholinesterase activities were determined by a micro version of the Ellman assay ${ }^{18}$ performed in a total volume of $100 \mu \mathrm{l}$ in microtitre plates, at $25^{\circ} \mathrm{C}, \mathrm{pH} 7 \cdot 0$. Acetylcholinesterase activities were measured at $\mathrm{V}_{\max }$ using acetylthiocholine $(1.0 \mathrm{mM})$ as substrate and distinguished from non-specific cholinesterase activity by use of the specific AChE inhibitor BW 284c51 $\left(1.5 \times 10^{-6} \mathrm{M}\right)$. Butyrylcholinesterase activities were also measured at $\mathrm{V}_{\max }$ using the substrate butyrylthiocholine $\left(1.5 \times 10^{-2} \mathrm{M}\right)$, which is not a substrate for AChE.

Protein content of CSF was determined using a micro version of the Biorad dye binding assay. ${ }^{19}$

STATISTICAL ANALYSIS

Data are presented as the mean (SEM). Results were analysed with Student's two tailed $t$ test and the Spearman rank correlation test.

\section{Results}

ACHE ACTIVITY IN CSF FROM PATIENTS WITH DEMENTIA

The mean AChE activity of ventricular CSF from patients with a histological diagnosis of Alzheimer's disease was $48 \%$ lower than that in age matched control patients (table 2). AChE activity in the ventricular CSF of patients with dementia of non-Alzheimer aetiology or with other neurological diseases was not significantly different from that in controls. A contrasting situation was found for the AChE activities of lumbar CSF: there was no significant difference between the AChE activity of CSF from patients with a histological diagnosis of Alzheimer's disease and that from age matched control patients, but AChE activity in CSF from patients with dementia of nonAlzheimer aetiology or with other neurological diseases was significantly lower than in control patients (table 2). An intermediate pattern was found in CSF sampled from the basal cistern: the AChE activity of CSF from patients with Alzheimer's disease or with dementia of nonAlzheimer aetiology was significantly lower than in control patients (table 2). AChE activity in the CSF of patients with the presenile form of Alzheimer's disease ( $<65$ years at onset) or Down's syndrome tended to be lower than in patients with the senile form 
Table 2 Mean (SE) acetylcholinesterase (AChE) activity (nmol/min/ml) of cerebrospinal fluid from different levels of the ventricular system of patients with dementia of the Alzheimer type

\begin{tabular}{|c|c|c|c|c|c|c|}
\hline \multirow[b]{2}{*}{ Patients } & \multicolumn{2}{|l|}{ Ventricular CSF } & \multicolumn{2}{|l|}{ Basal CSF } & \multicolumn{2}{|l|}{ Lumbar CSF } \\
\hline & $A C h E$ activity & $\%$ Decrease & $A C h E$ activity & $\%$ Decrease & $A C h E$ activity & $\%$ Decrease \\
\hline $\begin{array}{l}\text { Controls } \\
\text { Alzheimer's disease } \\
\text { Senile (onset after age 65) } \\
\text { Presenile (onset before age 65) } \\
\text { With Down's syndrome } \\
\text { Other types of dementia } \\
\text { Other neurological diseases }\end{array}$ & $\begin{array}{l}8 \cdot 2(1 \cdot 1)(n=18) \\
4 \cdot 3(0 \cdot 7) \star \star(n=19) \\
4 \cdot 9(0 \cdot 7)^{\star}(n=17) \\
0 \cdot 9(n=1) \\
2 \cdot 4(n=1) \\
7 \cdot 9(3 \cdot 5) n s(n=10) \\
8 \cdot 1(1 \cdot 4) n s(n=5)\end{array}$ & $\begin{array}{l}48 \\
40 \\
89 \\
71 \\
- \\
-\end{array}$ & $\begin{array}{l}20 \cdot 7(2 \cdot 1)(\mathrm{n}=19) \\
12 \cdot 5(1 \cdot 7)^{\star \star}(\mathrm{n}=17) \\
13 \cdot 8(1 \cdot 9)^{\star}(\mathrm{n}=13) \\
6 \cdot 7(\mathrm{n}=2) \\
9 \cdot 6(\mathrm{n}=2) \\
13.3(1 \cdot 6)^{\star}(\mathrm{n}=11) \\
20.5(6 \cdot 3) \mathrm{ns}(\mathrm{n}=5)\end{array}$ & $\begin{array}{l}40 \\
33 \\
68 \\
54 \\
36 \\
-\end{array}$ & $\begin{array}{l}16 \cdot 8(1 \cdot 5)(\mathrm{n}=13) \\
12.5(1 \cdot 4) \mathrm{ns}(\mathrm{n}=23) \\
13.4(1 \cdot 5) \mathrm{ns}(\mathrm{n}=20) \\
6.0(\mathrm{n}=2) \\
7.0(\mathrm{n}=1) \\
10.9(1 \cdot 8)^{\star}(\mathrm{n}=9) \\
9 \cdot 9(1 \cdot 1)^{\star}(\mathrm{n}=3)\end{array}$ & $\begin{array}{l}25 \\
20 \\
64 \\
58 \\
35 \\
41\end{array}$ \\
\hline
\end{tabular}

${ }^{\star} p<0.05,{ }^{\star \star} p<0.005$ compared with control patients.

of the disease, at all levels of the neuraxis from which CSF was collected (table 2).

AChE activity in CSF appeared unrelated to either the final cause of death or treatment with centrally acting drugs in the period before death.

Delay before postmortem examination was not significantly correlated with the $\mathrm{AChE}$ content of CSF from any of the three levels for control samples, (Spearman rank correlation coefficients of $0.029,0.161$, and 0.276 for ventricular, cisternal, and lumbar CSF respectively).

All the Alzheimer's disease patients had some degree of ventricular enlargement but this did not significantly affect the AChE content of ventricular fluid ( $p=0.69$, one way analysis of variance) with mean activities of $3 \cdot 12(1 \cdot 27) \mathrm{nmol} / \mathrm{min} / \mathrm{ml}$ for mild enlargement (four patients), $4.61(0.96) \mathrm{nmol} / \mathrm{min} / \mathrm{ml}$ for moderate enlargement ( 10 patients), and 4.59 $(1.41) \mathrm{nmol} / \mathrm{min} / \mathrm{ml}$ for severe enlargement (five patients).

CSF GRADIENTS OF ACHE ACTIVITY

Comparison of $\mathrm{AChE}$ activity from different
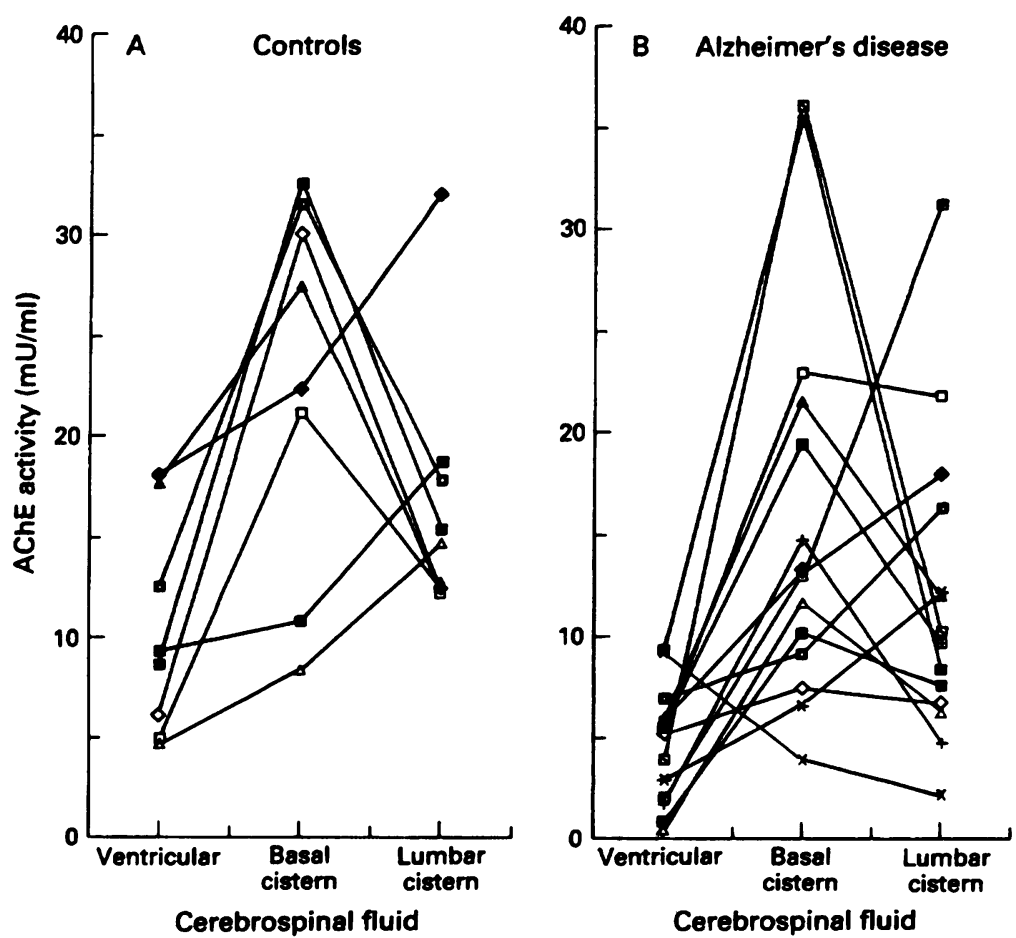

Figure 1 Acetylcholinesterase activity (hydrolysed acetylthiocholine, nmol/min/ml CSF) of CSF collected from three different levels of the neuraxis of (a) eight control patients and (b) 14 patients with dementia of Alzheimer type. levels of the neuraxis of control patients (from whom all three fluids were collected) showed that, in every case, AChE activity was higher in CSF taken from the basal cistern than in CSF taken from the ventricles $(p<0.01)$. However, activity in lumbar CSF was not significantly different from that in basal CSF (fig 1A). Findings were similar in CSF from patients with Alzheimer's disease (fig 1B).

BUCHE ACTIVITY IN CSF FROM PATIENTS WITH DEMENTIA

The mean BuChE activity of ventricular CSF from patients with Alzheimer's disease was significantly lower than that found in age matched control patients $(13.0(1.6)$ v 21.9 (2.0) $\mathrm{nmol} / \mathrm{min} / \mathrm{ml}$; $41 \%$ difference, $\mathrm{p}<0.05)$. BuChE activity in ventricular CSF from patients with dementia of non-Alzheimer aetiology $(27.4(5 \cdot 9)$, or from patients with other neurological diseases $(30.6(7 \cdot 0)$ was not different from that in control patients. There were no significant differences in $\mathrm{BuChE}$ activities of any group of patients when the CSF had been collected from the basal cistern or the lumbar cistern (data not shown).

The ratio of AChE:BuChE activity was similar in control patients and patients with Alzheimer's disease at each level of the CSF system, although there were rostrocaudal differences, with highest ratios being observed in CSF from the basal cistern (data not shown).

PROTEIN CONTENT OF CSF OF PATIENTS WITH DEMENTIA

The mean protein content of ventricular CSF from patients with Alzheimer's disease was significantly lower than that in age matched control patients $2.7(1.0)$ v $6.2(1.0) \mathrm{mg} / \mathrm{ml}$; $56 \%$ difference, $\mathrm{p}<0.05)$, whereas there was no significant difference in protein content of basal and lumbar CSF for these two groups of patients (data not shown).

\section{Discussion}

The AChE activity in samples of lumbar CSF obtained from control subjects at postmortem examination reported in this paper is comparable to that reported by other authors for activity in lumbar CSF obtained while patients were alive (hydrolysed acetylthiocholine, $8 \cdot 7-25.0 \mathrm{nmol} / \mathrm{min} / \mathrm{ml}){ }^{3561011}$ is Hence it would seem that change in AChE activity occurring after death is minimal, at least in lumbar CSF. AChE activity in CSF from the basal cistern was found to be consistently higher than in ventricular CSF from the same 
patient. This is in agreement with previous studies on rabbit CSF. $^{20}$ Postmortem delay was not correlated with the AChE activity of any of the three fluids from control patients, which also suggests that $\mathrm{AChE}$ activity is relatively stable after death.

The protein content of ventricular CSF samples from control patients is considerably higher than the normal protein content of ventricular fluid ${ }^{21}$ and indeed is higher than that previously recorded for postmortem ventricular CSF samples in our own laboratory. ${ }^{15}$ The latter difference could be attributed to the different assay techniques used (the earlier study used trichloroacetic acid precipitation and the Lowry assay); indeed, protein content of the CSF samples in this earlier study was higher if the trichloroacetic acid precipitation step was omitted (unpublished observation). This is probably due to the acid removing peptides that would otherwise contribute to the assay. However, the higher protein content of the ventricular fluid could be a result of the agonal condition of the subjects studied before and after death. The choroid plexus probably becomes more leaky after death and during the delay before collection of fluids, allowing plasma proteins and occasional red cells to pass into ventricular CSF and producing high protein concentrations. Such a process should not affect $A C h E$ in ventricular CSF since human plasma contains negligible AChE. The protein content of lumbar CSF in controls (5.4 $(1 \cdot 2) \mathrm{mg} / \mathrm{ml}$ ) was also higher than that previously reported by our laboratory for lumbar CSF obtained during life.

Our earlier studies of AChE activity in CSF from patients with a histological diagnosis of Alzheimer's disease produced results that differed according to the source of CSF. ${ }^{15}$ The results presented in this paper confirm these earlier findings: $\mathrm{AChE}$ in ventricular CSF from patients with Alzheimer's disease is much lower than normal, whereas lumbar CSF obtained from the same patients post mortem contains normal amounts of AChE. This suggests that the differences in ventricular and lumbar CSF are a real feature of the disease. Secretion of AChE from both forebrain and hindbrain regions seems greatly reduced in patients with Alzheimer's disease, leading to low activity of $\mathrm{AChE}$ from ventricular and basal cisternal CSF, while secretion from the spinal cord is unaffected (or even increased) so that it masks these changes, resulting in normal activity of $\mathrm{AChE}$ in the lumbar cistern.

Defective secretion of AChE also seems to be a feature of dementia of non-Alzheimer aetiology, but in these cases the defect seems to be confined to the hindbrain and spinal cord, resulting in reduced $\mathrm{AChE}$ activity in CSF from the basal and lumbar cisterns and normal ventricular levels. The deficit in AChE secretion in the hindbrain, which is common to both Alzheimer's and non-Alzheimer's dementias, may be responsible for some of the symptoms shared by these diseases. Such a disturbance of $\mathrm{AChE}$ secretion from the brain in dementia could affect not only cholinergic transmission in these patients but also the non- cholinergic modulatory actions that have been proposed for $\mathrm{AChE}^{2}$ For example, in the cerebellum at least, secreted AChE seems to enhance excitatory synaptic transmission mediated by amino acids. ${ }^{22}$

A more fundamental association between AChE and Alzheimer's disease has recently been suggested by the finding that purified AChE possesses a protease activity that can cleave the amyloid precursor protein (APP), ${ }^{23}$ so preventing the formation of the amyloidogenic BA4 protein, which is normally found in the plaques of Alzheimer's disease. Secreted AChE could therefore have an important role in the normal processing of APP at its cleavage site in the extracellular portion of the molecule; reduced secretions of AChE may therefore result in an increase in full length APP, encouraging abnormal processing to BA4 and deposition of amyloid.

The defect in secretion in Alzheimer's disease is probably not restricted to $\mathrm{AChE}$ since a similar situation was found for CSF protein content generally: content in ventricular CSF was greatly reduced in patients with $\mathrm{Alz}$ heimer's disease compared with age matched controls although protein concentrations in the basal and lumbar cisterns were in the normal range (confirming earlier reports from our laboratory $\left.{ }^{15}\right)$. An alternative explanation for the lowered ventricular concentrations of $\mathrm{AChE}$ and protein is that they are a result of dilution of the CSF due to the enlarged ventricular volume of Alzheimer's disease patient. ${ }^{25}$ However, AChE activity in ventricular CSF did not differ significantly in Alzheimer's disease patients with mild, moderate, or severe ventricular enlargement assessed post mortem, so this explanation seems unlikely.

Earlier reports from our laboratory suggested that BuChE was greatly reduced in both ventricular and lumbar CSF of Alzheimer's disease patients, resulting in a higher ratio of $\mathrm{AChE}$ to BuChE. ${ }^{18}$ We have, however, been unable to confirm all of these findings in the present group of patients: only in ventricular CSF was there a significant decrease in BuChE activity. The origin of BuChE in CSF is unclear, but high concentrations are present in blood plasma, hence variable degrees of plasma contamination of the CSF could occur after death and the breakdown of homeostasis as a result of deterioration of the blood-brain barrier; this would obscure any deficit.

Several authors have suggested that cholinesterase activities of CSF may be useful as a diagnostic tool in Alzheimer's disease. However, the results of the present study on patients with a histologically confirmed diagnosis suggest that $\mathrm{AChE}$ (and $\mathrm{BuChE}$ ) activities themselves would not be particularly useful in this respect, since only the ventricular CSF shows pronounced changes in AChE activity. It is neither practical, nor indeed ethically justifiable, to obtain ventricular CSF during life on a regular basis, purely for diagnostic purposes. Recent studies suggest, however, that the actual form of AChE secreted into CSF in patients with Alzheimer's 
disease is abnormal, displaying altered kinetic properties $^{15}$ and altered behaviour on isoelectric focusing, ${ }^{25}$ and this could provide the basis for a future diagnostic test using lumbar CSF.

B McD held a Wellcome research training fellowship in pathology; M E A held a Tilleard-Cole research fellowship. The work was supported by grants from the Grand Charity and from work was supported by grants from the Grand Charity and from Paul Butler for technical assistance during necropsies; $M$ Reading, S Lichfield, and R C Cross for neuropathological Reading, S Lichfield, and R C Cross for neuropathological and David Smith and Margaret Esiri for scientific discussions and advice.

1 Rakonczay Z. Cholinesterase and its molecular forms in pathological states. Progress in Neurobiology 1988 31:311-30.

2 Greenfield SA. A non-cholinergic action of acetylcholinesterase (AChE) in the brain: from neuronal secretion to the generation of movement. Cellular and Molecular Neurobiology 1991;11:55-77.

3 Arendt T, Bigl V, Walther F, Sonntag M. Decreased ratio of $\mathrm{CSF}$ acetylcholinesterase to butyrylcholinesterase activity in Alzheimer's disease. Lancet 1984;: i:173.

4 Tune L, Gucker S, Folstein M, Oshida L, Coyle JT. Cerebrospinal fluid acetylcholinesterase activity in senile dementia of

5 Nakano S, Kato T, Nakamura S, Kameyama M. Acetyl cholinesterase activity in cerebrospinal fluid of patients with Alzheimer's disease and senile dementia. $\mathcal{F}$ Neurol Sci 1986;75:213-23.

6 Atack JR, May C, Kaye JA, Kay AD, Rapoport SI Cerebrospinal fluid cholinesterases in aging and in dementia of the Alzheimer type Ann Neurol 1988;23:161-7.

7 Sirvio J, Kutvonen R, Soininen H, Hartikainen P, Reikkinen PJ. Cholinesterases in the cerebrospinal fluid, plasma and erythrocytes of patients with Alzheimer's disease. $₹$ Neural Transmission 1989;75:119-27.

8 Marquis JK, Volicer L, Mark KA, Direnfeld LK, Freedman $M$. Cholinesterase activity in plasma, erythrocytes and cerebrospinal fluid of patients with dementia of the Alzheimer type. Biological psychiatry 1985;20:605-10.

9 Bisso GM, Masullo C, Michalek H, Silveri MC, Pocchiar $M$. Molecular forms of cholinesterases in CSF of Alzheimer's disease/senile dementia of Alzheimer type patients and matched neurological controls. Life Sciences 1986;38:561-7.

10 Huff FJ, Maire JC, Growdon JH, Corkin S, Wurtman RJ.
Cholinesterases in cerebrospinal fluid: correlations with clinical measures in Alzheimer's disease. fournal of Neurological Science 1986;72:121-9.

11 Elble R, Giacobini E, Scarsella GF. Cholinesterases in cerebrospinal fluid: a longitudinal study in Alzheimer's disease. Arch Neurol 1987;44:403-7.

12 Ruberg M, Villageois A, Bonnet A, Pillon B, Reiger F, Agid $Y$. Acetylcholinesterase and butyrylcholinesterase activity in the cerebrospinal fluid of patients with neurodegenerative diseases involving cholinergic systems. $₹$ Neurol Neurosurg Psychiatry 1987;50:538-43.

13 Kumar V, Giacobini E. Cerebrospinal fluid choline and acetylcholinesterase activity in familial versus non-familial Alzheimer's disease patients. Archives of Gerontology ial Alzheimer's disease patien.
and Geriatrics 1988;7:111-17.

14 Khachaturian ZS. Diagnosis of Alzheimer's disease. Arch Neurol 1985;42:1097-105.

15 Appleyard ME, Smith AD, Berman P, Wilcock GK, Esiri MM, Neary D, et al. Cholinesterase activities in cerebrospinal fluid of patients with senile dementia of Alzheimer type. Brain 1987;110:1309-22.

16 Appleyard ME, Smith AD, Wilcock GK, Esiri MM Decreased CSF acetylcholinesterase activity in Alzheimer's disease. Lancet 1983;ii:452.

17 Appleyard ME, McDonald B. Acetylcholinesterase activities of cerebrospinal fluid from different levels of the central nervous system in patients with senile dementia of the Alzheimer type. Neurosci Lett 1990;(suppl 88):S114.

18 Appleyard ME, McDonald B, Benjamin L. Presence of a soluble form of acetylcholinesterase in human ocular soluble form of acetylcholinesterase in

19 Bradford MM. A rapid and sensitive method for the quantification of microgram quantities of protein utilising
the principle of protein-dye binding. Analytical Biothe principle of protein-dye

20 Greenfield SA, Chubb IW, Smith AD. The effect of chlorpromazine on the concentration of acetylcholinesterase in the cerebrospinal fluid of rabbits. Neuropharmacology 1979;18:127-32.

21 Davson H. Physiology of the cerebrospinal fluid. London: Churchill, 1967:272.

22 Appleyard ME, Jahnsen HJ. Non-cholinergic effects of acetylcholinesterase upon the membrane properties of mammalian cerebellar Purkinje cells. Eur $\mathcal{f}$ Neurosci 1989;(suppl 2):129.

23 Small DH, Moir RD, Fuller SJ, Michaelson S, Bush AI, Li $\mathrm{Q}$, et al. A protease activity associated with acetylcholinesterase releases the membrane-bound form of the amyloid terase releases the membrane-bound form of the amyloid
protein precursor of Alzheimer's disease. Biochemistry protein precursor

24 Soininen H, Puranen M, Reikkinen PJ. Computed tomography findings in senile dementia and normal aging. $f$ Neurol Neurosurg Psychiatry 1982;45:50-4

25 Navaratnam DS, Priddle JD, McDonald B, Esiri MM, Robinson JR, Smith AD. Anomalous molecular form of acetylcholinesterase in cerebrospinal fluid in histologically diagnosed Alzheimer's disease. Lancet 1991;i:447-9. 